\title{
USING FUNDAMENTAL PLANE DISTANCES to ESTIMATE the TOTAL BINDING MASS in ABELL 2626 ${ }^{1}$
}

\author{
Joseph J. Mohr \\ Departments of Physics and Astronomy, University of Michigan, Ann Arbor, MI 48109 \\ Gary Wegner \\ Department of Physics and Astronomy, Dartmouth College, Hanover, NH 03755 \\ Accepted for publication in The Astronomical Journal
}

\begin{abstract}
We use fundamental plane (FP) distance estimates to the components of the double cluster A2626 ( $c z \sim 17,500 \mathrm{~km} / \mathrm{s})$ to constrain cluster kinematics and estimate total binding mass. We employ deep $R$ band CCD photometry, multi-object spectroscopy, and software designed to account for seeing effects to measure the FP parameters $R_{e}$, $\sigma$, and $\left\langle\mu_{e}\right\rangle$ for 24 known early type and S0 cluster members. The FP coefficients from this sample $(\alpha=1.30 \pm 0.36$ and $\beta=0.31 \pm 0.06)$ are consistent with others reported in the literature.

We examine the $M g_{b}$ equivalent width distributions within both subclusters and find them to be indistinguishable. Lacking evidence for stellar population differences, we interpret the FP zeropoint offset between the two subclusters as a measure of the distance difference. We find $\log \left(D_{B} / D_{A}\right)=-0.037 \pm 0.046$, where $D_{c l}$ is the distance to subcluster $c l$. This measurement is consistent with the subclusters being at the same distance, and it rules out the Hubble flow hypothesis (distances proportional to velocity) with $99 \%$ confidence; analysis of the subcluster galaxy magnitude distributions rules out Hubble flow at $93 \%$ confidence. Both results favor a kinematic model where the subclusters are bound and infalling.

We estimate the total cluster binding mass by modelling the subcluster merger as radial infall. The projected separation, the line of sight velocity difference and the line of sight separation constrain the cluster mass; the minimum possible total binding mass is 1.65 times higher than the sum of the standard virial masses, a difference statistically significant at the $\sim 3 \sigma$ level. We discuss explanations for the inconsistency including (1) biases in the standard virial mass estimator, (2) biases in our radial infall mass estimate, and (3) mass beyond the virialized cluster region; if the standard virial mass is significantly in error, the cluster has an unusually high mass-to-light ratio $(\sim 1000 h)$. Because observational signatures of departures from radial infall are absent, we explore the implications of mass beyond the virialized, core regions.
\end{abstract}

\footnotetext{
${ }^{1}$ Observations reported here were obtained at the MDM Observatory, a facility jointly operated by the University of Michigan, Dartmouth College, and the Massachusetts Institute of Technology.
} 


\section{Introduction}

Galaxy cluster masses are clearly of cosmological significance, and examples abound: (1) the differences in thuster evolution as a function of mass provide constraints on the power spectrum Lacey \& Cole 1993, 1994), (2) comparisons of the amounts of luminous and non-luminous matter in clusters provide clues to the nature of dark matter and the efficiency of galaxy formation (David, Jones \& Forman 1995), and (3) the cluster mass-to-light ratio and baryon fraction constrain the cosmological density parameter $\Omega_{0}$ (e.g. Ramella, Geller \& Huchra 1989, White et al. 1993, Mohr et al. 1996).

Cluster mass studies tend to focus on the central regions where virial equilibrium is a more accurate approximation, and the weak lensing signals are easiest to detect. Here we apply a method to measure the mass beyond the virialized region in the double cluster A2626. We utilize recent improvements in the understanding of the fundamental plane (FP) to constrain the total cluster binding mass by revisiting the subcluster radial infall model (Beers, Geller \& Huchra 1982). Specifically, measuring FP distances (with an accuracy of $15 \%$ to $25 \%$ ) to a reasonably large sample of cluster galaxies can yield a very accurate (sub)cluster distance (Lucey \& Carter 1988, LyndenBell et al. 1988). For two bound subclusters, the line of sight separation, the line of sight velocity difference, and the projected separation provide enough information to estimate the total cluster binding mass in cases where a radial infall model is appropriate.

Although A2626 is rather distant, it is well suited for this analysis because simply demonstrating that the two subclusters are bound and infalling has interesting implications. A straightforward application of the radial infall model, using the (rest frame) line of sight velocity difference and the virial mass estimates within each subcluster, rules out bound and infalling merger models (Mohr, Geller \& Wegner 1996; hereafter MGW96); the virial masses are not large enough to produce the apparent velocity difference. Thus, FP distances used to determine whether or not the subclusters in A2626 are bound and infalling serve as an independent test of the virial estimators, or alternatively, as a means of measuring the mass beyond the virialized region of the cluster.

Possible pitfalls to this approach include (1) distance biasing FP zeropoint (or shape) differences between subclusters, (2) deviations from radial infall, and (3) significant interactions between the virialized regions of the two subclusters. FP variation with cluster environment is an area of active research (e.g. Guzman et al. 1992, Worthey, Trager, \& Faber 1996, Jørgensen, Franx \& Kjærgaard 1996; hereafter JFK96); current indications are that distance biases are smaller than $5 \%$ for ellipticals within clusters and are related to observable variations in the stellar populations within cluster galaxies. As discussed below, subcluster pairs suffering from pitfall numbers 2 and 3 can be identified through corresponding observational signatures. We argue that the radial infall model and FP distances provide a promising method for determining cluster masses on scales larger than the virialized region in low redshift clusters; if coupled with estimates of cluster light at larger radii, these mass measures should suffice to determine whether mass to light ratios vary significantly between the virialized core and the surrounding infall region. For higher redshift cluster pairs, our approach could be used to quantify the level of environmentally induced fundamental plane zeropoint variations.

We describe the double cluster A2626, the FP observations, and the reductions in $\S 2$. In Section 3, we derive FP coefficients, discuss constraints on the stellar populations in each subcluster, and derive constraints on their relative distances. Section 4 contains a discussion of the radial infall model applied to A2626. Conclusions are summarized in Section 5. Throughout the paper we use $H_{0}=100 \mathrm{~h} \mathrm{~km} / \mathrm{s} / \mathrm{Mpc}$.

\section{Galaxy Sample and Data}

Below we describe Abell 2626 Abell 1958, summarize previous observational results detailed elsewhere (MGW96), present the new observations, and describe the reductions and analysis in detail.

\subsection{Abell 2626}

A redshift survey with the Decaspec (Fabricant \& Hertz 1990 mounted on the MDM Hiltner $2.4 \mathrm{~m}$ telescope in Fall 1993 and 1994 revealed that Abell 2626 $(\langle c z\rangle \sim 17,500 \mathrm{~km} / \mathrm{s}$, richness class 0$)$ is composed of at least two systems with mean line of sight velocities which differ by $\sim 2,500 \mathrm{~km} / \mathrm{s}$ (MGW96). As is clear from Fig. 1 and Table 1, there are two main components with cluster-like dispersions: group A centered at a velocity of $\langle c z\rangle=16,533 \pm 141 \mathrm{~km} / \mathrm{s}$ and group $\mathrm{B}$ centered at a velocity of $\langle c z\rangle=19,164 \pm 138 \mathrm{~km} / \mathrm{s}$ (90\% statistical confidence limits). As discussed in 
MGW96, the 11 galaxies centered at $\langle c z\rangle=21,173 \pm$ $119 \mathrm{~km} / \mathrm{s}$ are most likely part of a background, low density structure because (1) the dispersion is low $\left(\sigma=200_{-52}^{+119} \mathrm{~km} / \mathrm{s}\right)$ and (2) a large fraction of these galaxies are gas rich - $73 \%$ have emission lines compared to $39 \%$ (33\%) for group A (B). If the velocity of the low density structure corresponds to Hubble flow then its distance from groups A and B is from $20 h^{-1} \mathrm{Mpc}$ to $50 h^{-1} \mathrm{Mpc}$.

The group A and B masses, velocity dispersions, central densities and X-ray properties all differ. As noted in Table 1, the virial mass of subgroup $\mathrm{A}$ is roughly twice that of $\mathrm{B}$, and the line of sight velocity dispersions are $\sigma_{A}=658_{-81}^{+111} \mathrm{~km} / \mathrm{s}$ and $\sigma_{B}=$ $415_{-72}^{+117} \mathrm{~km} / \mathrm{s}$ (90\% statistical confidence limits). The absence of observed X-ray emission from group B can be used to place a limit on the central gas density in group B (MGW96). In particular, the Einstein imaging proportional counter (IPC) image of the region reaches roughly 50 times fainter than the peak in the $\mathrm{X}$-ray emission from group $\mathrm{A}$. The group $\mathrm{A}$ central gas density is $\sim 3 \times 10^{-3} \mathrm{~cm}^{-3}$, so the central gas density in group $\mathrm{B}$ must be $<5 \times 10^{-4} \mathrm{~cm}^{-3}$. Although the contrast in the projected galaxy densities of groups A and B is difficult to quantify, the smoothed distribution (Figure 3 in MGW96) indicates that the central density in group B is $\sim 7$ times less than the central density in group A. Thus, the ratio of gas to galaxy density may be the same in both groups.

As discussed below, if groups A and B are on a radial infall trajectory, then the minimum implied gravitational mass is more than the sum of their virial masses. However, it is also possible that the velocity difference is just due to Hubble flow. The magnitude distributions of the confirmed members of both groups indicate that the merger hypothesis is favored over Hubble flow at $93 \%$ confidence (MGW96). To further investigate this issue we measure fundamental plane distances to each cluster and determine the line of sight distance difference directly. To do so we first use $R$ band CCD photometry to identify a sample of early type galaxies with redshift confirmed membership.

\subsection{Photometry}

On November 21, 1995, we used a thinned, Tektronix $1024^{2}$ CCD mounted on the MDM Hiltner $2.4 \mathrm{~m}$ telescope to image galaxies in A2626 and J8
(Jackson 1982). The $24 \mu$ pixels are 0.275 " on a side. The seeing varied during the night between 0.85 " and 1.05 ". Because the night was non-photometric, we determine the photometric zeropoint of each $R$ band image externally. For the A2626 galaxies we use photometric images taken previously (MGW96) on the MDM $1.3 \mathrm{~m}$ to zeropoint our images. Specifically, we use aperture photometry of isolated stars in the $2.4 \mathrm{~m}$ and $1.3 \mathrm{~m}$ frames to determine the $2.4 \mathrm{~m}$ zeropoint. The $1.3 \mathrm{~m}$ images are reduced to the Kron-Cousins system using Landolt (1992) standards. For J8 we rely primarily on published photoelectric galaxy aperture photometry (Colless et al. 1993) to determine the $\mathrm{R}$ band zeropoint but also use stellar aperture photometry from a photometric $1.3 \mathrm{~m}$ image for one galaxy (Saglia et al. 1997). In addition to our primary imaging run, we acquired additional Hiltner $2.4 \mathrm{~m}$ images through the generosity of Paul Schechter; the reductions for these data were similar.

The $R$ band galactic extinction is $0.062 \mathrm{mag}$ toward A2626 (NASA/IPAC Extragalactic Database, Savage \& Mathis 1979) and $0.185 \mathrm{mag}$ toward J8 Saglia et al. 1997). We approximate the $k$ correction as $k_{R}=z_{g}$ where $z_{g}$ is the galaxy redshift Frei \& Gunn 1994). We account for the peculiar velocity component of the line of sight velocity when applying the cosmological dimming correction; specifically, two of the factors of $(1+z)$ in the cosmological dimming are due to relativistic effects and two are due to the change in the geometry of the universe between emission and detection. Writing $z_{g}=z_{d}+z_{p}$, where $z_{d}$ and $z_{p}$ are the Hubble flow and peculiar velocity components of the redshift, the cosmological dimming is written $C=+5 \log \left\{1+z_{d}\right\}+5 \log \left\{1+z_{g}\right\}$; in terms of the observed and peculiar redshift, the correction is $C=+10 \log \left\{\left(1+z_{g}\right) \sqrt{1-z_{p} /\left(1+z_{g}\right)}\right\}$. Thus, $C$ is model dependent. As an example, if groups A and $\mathrm{B}$ are at the same distance and the peculiar velocity correction were incorrectly taken to be $z_{p}=0$, the fundamental plane distances would be biased so that group B would appear closer than group A by $\sim 1 \%$.

We bias subtract, flat field (using twilight flats), and clean the images using standard IRAF tasks. For each galaxy, we calculate sky subtracted radial profiles and uncertainties. Uncertainties have a Poisson component added in quadrature with an assumed $1 \%$ flat fielding uncertainty. We fit these profiles to summed $R^{1 / 4}$ laws and exponential disks,

$$
I_{b}(R)=I_{b 0} e^{-7.67\left[\left(R / R_{b}\right)^{1 / 4}-1\right]}
$$




$$
I_{d}(R)=I_{d 0} e^{-R / R_{d}}
$$

where $I_{b 0}\left(R_{b}\right)$ and $I_{d 0}\left(R_{d}\right)$ are the characteristic surface brightnesses (scale lengths). Our fitting procedure accounts for the effects of PSF smoothing and pixel extent as described in Saglia et al. 1993b.

More specifically, we determine the parameters of the $R^{1 / 4}$ and disk components by minimizing the $\chi^{2}$ difference between the seeing convolved model and the observed profile. The expected surface brightness $I(R, d R)$ in the bin of width $2 d R$ and radius $\mathrm{R}$ is

$$
I(R, d R)=\frac{[F(R+d R)-F(R-d R)]}{4 \pi R d R}
$$

where $F(R)$ is the seeing convolved integral flux within radius $R$. The integral flux follows from the bulge, disk and PSF parameters. Specifically,

$$
F(R)=R \int_{0}^{\infty} d k J_{1}(k R) \hat{p}(k)\left(\hat{I}_{b}(k)+\hat{I}_{d}(k)\right)
$$

where $J_{1}$ is a Bessel function, and $\hat{p}(k), \hat{I}_{b}(k)$, and $\hat{I}_{d}(k)$ are the Fourier transforms of the PSF, the bulge, and the exponential disk. Following Saglia et al. $1993 \mathrm{~b}$, we use a PSF of the form

$$
\hat{p}(k)=e^{-(k b)^{\gamma}}
$$

and find it to be a good fit to stars on the $2.4 \mathrm{~m}$ images with $1.4 \leq \gamma \leq 1.6$. We use the analytic approximation to the Fourier transform of the $R^{1 / 4}$ law (Saglia et l. 1993b), and the exact form of the transform of the disk component (e.g. Bracewell 1986).

The fitting procedure has three steps:

- Fit the PSF parameters $b$ and $\gamma$ using radial profiles of $\sim 4$ stars within each $2.4 \mathrm{~m}$ image; best fit parameters minimize the $\chi^{2}$ difference between the observed and model profile.

- Feed initial guesses for the bulge and disk parameters into a simplex minimization routine (Press et al. 1988) which minimizes the $\chi^{2}$ difference between the seeing convolved theoretical and observed profiles. At each iteration the full Fourier integral is solved for each point in the radial profile; with an $\mathrm{HP}-735$ the minimization takes several minutes. In addition to the uncertainties in the observed profile, a theoretical uncertainty is introduced to account for imperfections in the smoothing corrections (e.g. the exact form of the PSF). Specifically, the uncertainty for each profile point has a component which is proportional to the fractional effect of the smoothing on the model profile. This has the positive effect of making the fit results less sensitive to the inclusion/exclusion of the heavily smoothed inner points in the profile and to the details of the smoothing operation. We include a theoretical uncertainty which is $10 \%$ of the fractional effect of the smoothing on the model profile. This is consistent with a philosophy that we can correct for seeing effects at the $90 \%$ level.

- We fit a bulge only model to the profile and compare the minimum $\chi^{2}$ for this model to the bulge plus disk $\chi^{2}$. For galaxies where $\chi^{2}$ is only minimally improved by the inclusion of the disk component we use the bulge only fit. Once the fitting is complete, we use the best fit parameters to calculate $R_{e^{-}}$the half luminosity radius, $\mu_{e}$ - the surface brightness at $R_{e}$, and $\left\langle\mu_{e}\right\rangle$ - the mean surface brightness within $R_{e}$.

We test our fitting routines by (1) fitting artificial galaxy images, (2) comparing results from multiple images of the same galaxy, and (3) comparing our results to those of Saglia et al. (1997) in the cluster J8. We create artificial images by adding a galaxy described by an $R^{1 / 4}$ profile to a flat background of $10^{3} \mathrm{cts} /$ pixel. We then smooth the artificial image and introduce Poisson noise. By using delta functions instead of $R^{1 / 4}$ profiles we produce stars which are then used to measure the PSF parameters $b$ and $\gamma$. The process of fitting artificial galaxy images tests the accuracy of our fitting in that case where the true galaxy profile is well described by an $R^{1 / 4}$ profile. We find that the parameters $R_{e}$ and $\left\langle\mu_{e}\right\rangle$ are constrained to an accuracy of $\Delta \log \left(R_{e}\right) \sim 0.02$ and $\Delta\left\langle\mu_{e}\right\rangle \sim 0.07$ for $R_{e}$ as small as $50 \%$ the FWHM of the PSF. As expected, the accuracy of the combination $\log \left(R_{e}\right)-0.33 *\left\langle\mu_{e}\right\rangle$ (which appears in the equation describing the fundamental plane) is constrained to $\sim 0.01$ because the errors in $R_{e}$ and $\left\langle\mu_{e}\right\rangle$ are correlated.

We also compare results from subarcsecond seeing images of seven A2626 galaxies to results from poorer quality images. The mean offset between the parameters in the good and poor seeing images of the same galaxies are consistent with zero, and the scatter is small. Specifically, $\Delta\left\langle\mu_{e}\right\rangle=-0.0262 \pm 0.0311$ with 
an RMS of $0.0823, \Delta \log \left(R_{e}\right)=-0.0073 \pm 0.0074$ with an RMS of 0.0196 , and $\Delta\left(\log \left(R_{e}\right)-0.33 *\left\langle\mu_{e}\right\rangle\right)=$ $0.0013 \pm 0.0037$ with an RMS of 0.0097 .

We observed five galaxies in the EFAR cluster J8 (Wegner et al. 1996) for which we have photometric zeropoints. These observations provide the strongest test of our methods because the data, reductions and analyses differ significantly. There are no significant offsets between our values and EFAR values (Saglia et al. 1997); specifically, $\Delta\left\langle\mu_{e}\right\rangle=-0.0766 \pm 0.1223$ with an RMS of $0.2735, \Delta \log \left(R_{e}\right)=-0.0257 \pm 0.0326$ with an RMS of 0.0729 , and $\Delta\left(\log \left(R_{e}\right)-0.33 *\left\langle\mu_{e}\right\rangle\right)=$ $-0.0006 \pm 0.0112$ with an RMS of 0.0249 . These three tests of our photometric methods indicate that our procedure is accurate and makes only a small contribution to the observed scatter in the fundamental plane (see below).

\subsection{Spectroscopy}

On November 22 and 23, 1995, we used the Decaspec (Fabricant \& Hertz 1990) plus MkIII spectrograph mounted on the MDM Hiltner $2.4 \mathrm{~m}$ telescope to obtain spectra of four galaxy fields in Abell 2626, one field in J8 (Wegner et al. 1996), and multiple spectra of five HD stellar velocity standards. The Decaspec fibers subtend $2.3^{\prime \prime}$ in the focal plane. We used a grism blazed at $5.700 \AA$ with $600 \mathrm{l} / \mathrm{mm}$. vielding a typlical PSF of $5.3 \AA$ at $2.18 \AA$ per pixel and coverage from $4,300 \AA$ to $6,500 \AA$. Four or five thirty minute exposures were obtained for each of the A2626 fields; shorter exposures were obtained for the brighter J8 field. We extracted the spectra, using arc lamp exposures taken on either side of each object exposure to dispersion correct. We then combined the spectra from the four to five exposures, excluding cosmic rays through sigma clipping of large, positive deviations. With the Decaspec there are four sky spectra for each object spectrum. We combined the sky spectra for each object, scaled the sky spectrum using the flux in the $5577 \AA$ sky line, and then removed the sky contribution from the object spectrum. The final spectra range in signal to noise $(\mathrm{S} / \mathrm{N})$ from $\mathrm{S} / \mathrm{N}=20$ to 75 per pixel at $5,300 \AA$.

We obtained spectra of more galaxies, stellar velocity and Lick linestrength standards Worthey et al. 1994) using the Modspec mounted on the MDM Hiltner $2.4 \mathrm{~m}$ telescope in Nov and Dec '96. We used a $1,200 \mathrm{l} / \mathrm{mm}$ grating, the $200 \mathrm{~mm}$ camera, a thinned CCD with $24 \mu \mathrm{m}$ pixels, and a 1.7" wide slit; standard longslit reductions led to spectra with $2 \AA$ reso- lution, $1 \AA$ /pixel sampling, and coverage from $4,800 \AA$ to $5,800 \AA$. Galaxy exposure times ranged from $20 \mathrm{~min}$ to $2.5 \mathrm{hrs}$, and the spectra have $\mathrm{S} / \mathrm{N} \geq 30$ per pixel. The higher resolution of this setup allowed us to accurately measure galaxy dispersions as low as $100 \mathrm{~km} / \mathrm{s}$.

For each run, galaxy dispersions and velocities are extracted using the stellar templates from that run and the cross correlation peak fitting available in $\mathrm{fx}$ cor (IRAF.RV); we use the approximate wavelength region $4,800-5,800 \AA$ which contains the strong $\mathrm{Mg}$ features. The correlation peak width is transformed to an intrinsic galaxy dispersion using translation tables created for each stellar template; the translation tables are produced by cross correlating the stellar templates against Gaussian broadened versions of themselves. The final galaxy dispersion is the mean of the dispersions from each stellar template in the run. Finally, an aperture correction

$$
\log \left(\frac{\sigma_{c o r}}{\sigma_{o b s}}\right)=0.038 \log \left(\frac{r_{a p}}{r_{a p}^{n}} \frac{\theta_{e}^{n}}{\theta_{e}}\right)
$$

is applied. This aperture correction accounts for the falling dispersion profile in early type galaxies; we normalize to an aperture of radius $r_{a p}^{n}=1.7^{\prime \prime}$ and a galaxy with $\theta_{e}^{n}=20^{\prime \prime}$. For the longslit spectra we use $r_{a p}=1.025 \sqrt{x y / \pi}$, where $x$ and $y$ are the width and length of the rectangular aperture (Jørgensen, Franx \& Kjærgaard 1995, Baggley 1996).

We estimate the uncertainties in our dispersions using Monte Carlo techniques. For each galaxy spectrum we broaden a stellar template to mimic the measured dispersion, scale the template to the observed cts/pix in the region around 5,200 $\AA$, and add the sky at the observed level. We sample this artificial galaxy spectrum 100 times, introducing the Poisson noise and sky subtracting, and then measure the dispersion. We use the RMS of the measured dispersions around the input value as a measure of the velocity dispersion uncertainty (see Table 2). Not surprisingly, the simulations indicate that averaging the dispersions measured by cross correlating against multiple templates does not improve the accuracy. However, these simulations consider only Poisson noise effects; we average the multiple dispersion measurements from each galaxy spectrum to reduce template mismatch systematics.

We have multiple spectra for 21 galaxies. The distribution of dispersion differences scaled by the uncertainties for the 28 comparison pairs has an RMS of 0.97, indicating that the Monte Carlo uncertain- 
ties are a reasonably good estimate of our true dispersion uncertainties. The mean scaled difference $\left(\left\langle\left(\sigma_{M o d}-\sigma_{\text {Deca }}\right) / \epsilon_{\sigma}\right\rangle\right.$ where $\epsilon_{\sigma}$ is the uncertainty in the difference) between Modspec and Decaspec measurements for 23 comparison pairs has a mean of $-0.11 \pm 0.19$ with an RMS scatter about this mean of 0.89 ; the variance weighted, mean logarithmic difference between Modspec and Decaspec dispersions is $-0.001 \pm 0.013$. Because there is no evidence for a systematic difference between dispersions measured with the Decaspec and the Modspec, we apply no correction. We note that the Modspec was used in measuring dispersions for 10 of the 16 galaxies in group $\mathrm{A}$ and 5 of the 8 galaxies in group B; so any systematic offset between Decaspec and Modspec measurements would add to the FP scatter within each group, but would not bias the estimate of the distance difference.

Table 2 contains a list of the galaxies and their properties; for the galaxies with multiple measurements, we use the variance weighted average dispersion. The columns of Table 2 contain the galaxy tag (first letter corresponds to group membership), the coordinates, the redshift $c z$, the velocity dispersion $\sigma$ and uncertainty $\epsilon_{\sigma}$, the $R$ band apparent magnitude $M_{R}$, the mean surface brightness $\left\langle\mu_{e}\right\rangle$ within the half light radius, the half light radius $\theta_{e}$, the $M g_{b}$ equivalent width, and the bulge luminosity fraction $F_{B}$.

For 6 galaxies in J8 we compare our measured dispersions with those of Saglia et al. (1997). There is evidence of an offset; the distribution of $\left(\sigma_{E F A R}-\right.$ $\left.\sigma_{o b s}\right) / \epsilon_{\sigma}$ has a mean of $-0.80 \pm 0.31$ (where $\epsilon_{\sigma}$ is the uncertainty in the dispersion difference). The average EFAR velocity dispersion is $15 \pm 7 \mathrm{~km} / \mathrm{s}$ lower than ours. This offset does not affect our estimates of the relative distances to the two subclusters, but would complicate efforts to bring our distances onto the EFAR system.

We place constraints on possible differences in the stellar populations of the two subclusters by using IRAF scripts to measure $M g_{b}$ linestrengths in each galaxy spectrum. The rest wavelengths given in Burstein et al. (1984) for the feature and continuum bands are corrected using the redshifts in Table2. We broaden our spectra to the nominal $8.6 \AA$ resolution of the Lick system (Worthey \& Ottaviani 1997) before making measurements. We tranform to the Lick system using an expression of the form

$$
M g_{b}^{L I C K}=a f(\sigma) M g_{b}^{o b s}+c_{a p},
$$

where $a$ is a scale factor, $f(\sigma)$ is a velocity disper- sion correction we determine by broadening our Lick standards, and $c_{a p}$ is an aperture correction of the same form as in Eq. 2-5 but with a coefficient of 0.050 instead of 0.038 . We determine $a$ separately for each run; observations of Lick standards indicate $a=1.060 \pm 0.016$ for the Nov' 96 and $a=1.086 \pm 0.028$ for the Dec '96 data. We use overlapping galaxy observations to measure $a=1.066 \pm 0.034$ for the Nov '95 data. The aperture correction is similar to that used by Jørgensen (1997), but generalized to the form used for the velocity dispersions above Baggley 1996).

We test the accuracy of our $M g_{b}$ measurements using multiple galaxy observations. Twenty three comparison sets from 18 different galaxies yield an RMS of 0.051 in $\Delta \log M g_{b}$. Thus, we estimate the uncertainty of a single observation is approximately $9 \%$. The linestrengths listed in Table 2 are averages of multiple observations where appropriate. Finally, we compare our linestrengths to preliminary EFAR $M g_{b}$ linestrengths in $6 \mathrm{~J} 8$ cluster galaxies; the EFAR values are lower: $\Delta \log M g_{b}=-0.0733 \pm 0.014$ with an RMS of 0.039. The linestrengths in Table 2 are corrected to the EFAR system.

\section{Fundamental Plane Analysis}

In this section we, determine the coefficients and zeropoint of the FP within the two clusters (§3.1), discuss the evidence for stellar population variations (§3.2), and then interpret the observed FP zeropoint offset (§3.3).

\subsection{Determining FP Coefficients}

Even if the assumption that elliptical and S0 galaxies within both clusters are similarly distributed within the FP is valid, the FP coefficients and cluster distances can be biased by differences in selection (e.g. Lynden-Bell et al. 1988, Baggley 1996). Our galaxy sample is drawn from a list of known elliptical and S0 cluster members sorted by central aperture magnitude (aperture is $2^{\prime \prime} \times 2^{\prime \prime}$ square); the parameters of our final sample indicate that central aperture magnitude is correlated with $\log \theta_{e}-0.3\left\langle\mu_{e}\right\rangle$, a quantity similar to the combination which appears in the FP. Thus, our selection is similar to selection by isophotal diameter $D_{n}$ (Dressler et al. 1987). We seek to minimally bias the coefficients by (1) combining the samples from both clusters into a single FP fit and (2) weighting galaxies within each cluster by the sam- 
ple completeness. Finally, we examine the variation of the zeropoint offset between the two clusters as a function of variations in the FP coefficients ( $\$ 3.3)$.

Our galaxy sample extends over $1.5 \mathrm{mag}$ in central aperture magnitude. We divide this range into four bins and calculate the completeness within each separately for the two clusters. The completeness in each bin is simply the number of galaxies within our final FP sample divided by the estimated total population of elliptical and S0 cluster members. We estimate the total cluster population within each bin to be $N_{c l}=N_{t o t} * f_{c l}$, where $N_{t o t}$ is the total number of elliptical and S0 galaxies identified in the $R$ band survey of the cluster, and $f_{c l}$ is the fraction of all elliptical and S0 galaxies with redshifts that lie within the velocity range of cluster $c l$.

Weighting by the inverse of the completeness appears to be ill advised for the faintest galaxies within each cluster. For these two galaxies the completeness is s mall enough (weight is 15.2 (17) for the faimtest in group A (B)), that weighting by the incompleteness woptld lead to them dominating the fit. We exclude the faimtest galaxy from each sample when determining the FP coefficients, but include them when calculating the best estimate of the cluster zeropoint. Finally, we normalize the weights within each cluster so that the ratio of weighting for cluster $\mathrm{A}: \mathrm{B}$ is $15: 7$, the ratio of the number of galaxies in each.

We fit the galactic properties to a $\mathrm{FP}$ of the form $\log \theta_{e}=\alpha \log \sigma+\beta\left\langle\mu_{e}\right\rangle+\gamma$, where $\alpha$ and $\beta$ are the (cluster invariant) coefficients of the plane and $\gamma$ is a distance dependent zeropoint (Faber et al. 1987, Djorgovski \& Davis 1987, Jørgensen, Franx \& Kjærgaard 1993, JFK96). We combine galaxies from both groups in a single fit by using deviations around median values of distance dependent quantities (Baggley 1996). The corrected quantities $\left\langle\mu_{e}\right\rangle$ and $\log \sigma$ are distance independent, so we transform $\log \theta_{e}$ alone; specifically, we remove an estimate of the zeropoint within each subcluster by expressing $\log \theta_{e}$ as the variation around the $\gamma_{f i t}$ which minimizes the cluster scatter in $\gamma$

$$
\log \theta_{e} \rightarrow \log \theta_{e}-\gamma_{f i t}
$$

Note that the transformation depends on the FP coefficients, so it must be reapplied during each fit iteration.

We determine the best fit FP coefficients by minimizing the sum of the absolute value of the weighted, orthogonal deviations from the plane (JFK96, Baggley 1996). The best fit values are $\alpha=1.30$ and $\beta=0.31$; the scatter in $\log R_{e}$ is 0.090 (23\% distance uncertainty). Our FP has the form

$$
\log \theta_{e}=(1.30 \pm 0.36) \log \sigma+(0.31 \pm 0.06)\left\langle\mu_{e}\right\rangle+\gamma
$$

The uncertainties are determined using a bootstrap resampling procedure (JFK96, Baggley 1996) to resample the galaxy list and fit the FP $10^{4}$ times; the uncertainty intervals are half the width of the $68 \%$ confidence region. For comparison, the FP coefficients in the case where all galaxies are given equal weight are $\alpha=1.27$ and $\beta=0.27$. Our best fit FP coefficients are statistically consistent with the values $\alpha=1.24 \pm 0.07$ and $\beta=0.33 \pm 0.01$ determined in a sample of 207 galaxies with Gunn $r$ photometry (JFK96); as discussed by JFK96, their coefficients are consistent with previous studies (e.g. Faber et al. 1987, Djorgovski \& Davis 1987, Bender, Burstein \& Faber 1992, Guzman, Lucey \& Bower 1993, Saglia, Bender \& Dressler 1993, Jørgensen, Franx \& Kjærgaard 1993). Fig. 2 contains plots of the group A (filled) and B (hollow) galaxies along two projections of the FP; the lines in each figure represent the FP for groups A (solid) and B (dashed).

\subsection{Constraints on the Stellar Populations}

We compare the distribution of $M g_{b}$ equivalent widths within the two subclusters to place constraints on stellar population variations which could introduce offsets in the cluster FP zeropoints, biasing the relative distances to the two clusters (e.g. JFK96). Fig. 3 contains a plot of $\log \left(M g_{b}\right)$ versus $\log \sigma$ for the galaxies in groups A (solid points) and B (hollow points). The lines represent best fit models for groups A (solid) and $\mathrm{B}$ (dashed).

Rather than allowing both the slope and zeropoint to vary, we constrain the slope to be that found by Jørgensen (1997) for a sample of $\sim 300$ galaxies. Specifically, the combined correlations of $M g_{2}$ with $M g_{b}$ and $M g_{2}$ with $\sigma$ imply

$$
\log M g_{b}=0.307 \log \sigma-b
$$

where the zeropoint for the large galaxy sample is $b=0.034$ (Jørgensen 1997). By minimizing orthogonal deviations from the line, we find $b=0.054 \pm 0.016$ for sample A and $b=0.042 \pm 0.022$ for sample B. The zeropoint of each cluster is statistically consistent with the zeropoint from the Jørgensen sample. We 
can measure the offset between the two clusters somewhat more accurately; the zeropoint difference between the two clusters is $\Delta b=b_{A}-b_{B}=0.012 \pm 0.019$, statistically consistent with no offset.

Trends in $M g_{2}$ have been noted as a function of radius from the center of the Coma cluster Guzman et l. 1992) and as a function of cluster velocity dispersion for a sample of 11 clusters (JFK96; Jørgensen 1997). Another study indicates that elliptical galaxies in low velocity dispersion environments have both higher metallicities and younger stellar populations than galaxies in higher velocity dispersion environments (Rose et al. 1994). The velocity dispersion difference between clusters A $(\sigma=658 \mathrm{~km} / \mathrm{s})$ and $\mathrm{B}(\sigma=415 \mathrm{~km} / \mathrm{s})$ should introduce an offset of $\Delta b \sim-0.016$ into the $M g_{b}-\sigma$ correlation. This expectation is inconsistent with our observations at the $1.5 \sigma$ level; we would require additional galaxy linestrengths to resolve an offset of that magnitude.

\subsection{FP Zeropoint Differences}

We determine the zeropoint of each group by using the median $\gamma$; the 16 galaxies in group A yield $\gamma_{A}=$ -8.4083 with an RMS around this value of 0.0879 , and the 8 galaxies in group $\mathrm{B}$ yield $\gamma_{B}=-8.3632$ with an RMS of 0.1149 . The homogeneous Malmquist bias has a marginal effect on the relative distances to these two clusters (Lynden-Bell et al. 1988); we calculate $\delta \gamma_{A}=-0.0033$ and $\delta \gamma_{B}=-0.0114$, resulting in an increase in $\Delta \gamma$ of $\sim 0.0081$. Thus, the zeropoint difference between the two clusters is

$$
\Delta \gamma=\gamma_{A}-\gamma_{B}=-0.037 \pm 0.046
$$

where we have assumed the uncertainty in the zeropoint is the scatter about that value divided by $\sqrt{N}$, where $N$ is the number of galaxies (note that this is a conservative estimate of the true uncertainties because the scatter is sensitive to outliers). As discussed in $\S 2.2$, if we assume groups A and B are at the same distance rather than at distances proportional to their mean redshifts, the cosmological dimming corrections change so that $\Delta \gamma$ increases by $\sim 0.004$.

In general, zeropoint offsets can be caused by stellar population or metallicity induced $M / L_{R}$ differences and/or distance differences. Because there is no compelling evidence for stellar population differences, we use the observed zeropoint offset and uncertainty as relative distance constraints. The quantity $\gamma$ (in Eqn. 3-2) can be written $\gamma_{x}=\log \left(\Gamma / D_{x}\right)$ where $D_{x}$ is the distance of galaxy $x$ and $\Gamma$ follows from the standard form implied by the FP

$$
R_{e}=\Gamma \sigma^{\alpha}\left\langle I_{e}\right\rangle^{-2.5 \beta}
$$

Assuming the intrinsic FP zeropoint $\log \Gamma$ is the same for all galaxies, the relative distances to two galaxies $x$ and $y$ is

$$
\log \left(\frac{D_{x}}{D_{y}}\right)=\gamma_{y}-\gamma_{x} .
$$

Fig. A contains a plot of the distances to the galaxies in groups A (solid) and B (hollow) relative to the median distance to group A versus the galaxy redshift. Also marked with vertical lines are the (homogeneous Malmquist bias corrected) median distances to groups A $\left(D_{A} ;\right.$ solid line $)$ and $\mathrm{B}\left(\log \left(D_{B} / D_{A}\right)=-0.037\right.$; dashed line); the horizontal lines mark the mean velocities for groups A (solid) and B (dashed). The error bars on the dashed, vertical line represents the relative distance uncertainty. The large star marks the position of cluster $\mathrm{B}$ if its distance relative to cluster $\mathrm{A}$ were reflective of pure Hubble flow $(\Delta D \propto \Delta\langle v\rangle)$; the Hubble flow hypothesis implies $\log \left(D_{B} / D_{A}\right)=0.065$.

The zeropoint difference is consistent with zero, and it is sufficient to rule out Hubble flow at $2.2 \sigma$; therefore, the FP measurements suggest that cluster $\mathrm{B}$ is closer to us than cluster A (in $\S 5.1$ we return to this issue in more detail). Naturally, the zeropoint offset depends on the coefficients of the FP, which are only constrained to within $\sim 20 \%$ of the best fit values. Using the distribution of $\gamma_{A}-\gamma_{B}$ from the $10^{4}$ bootstrap resampling fits, we find that in $99.05 \%$ of the simulations the zeropoint offset between the two clusters is less than the Hubble flow offset, rejecting the Hubble flow hypothesis. This probability corresponds to a $+2.35 \sigma$ deviation in a Gaussian, similar to the $2.2 \sigma$ estimate above which we derived from the scatter about the median distance in each cluster. The bootstrap probability is superior because it includes variations in the FP coefficients (and is less sensitive to outliers than the scatter estimator).

\section{Radial Infall Model as a Mass Constraint}

The radial infall model has been applied to many close galaxy cluster pairs to determine whether or not the pairs will merge (e.g. Beers, Geller \& Huchra 1982, Beers et al. 1991, Beers et al. 1992, Colless \& Dunn 1995, Scodeggio et al. 1995). In the following we use the measured line of sight separation and this model to estimate the total binding mass required to 
explain the observed infall velocity. We then compare this mass to virial estimates of the cluster binding mass.

\subsection{Radial Infall Model}

For two isolated, bound objects within an expanding universe, the dynamics can be parametrized in the standard way

$$
\begin{array}{r}
t=B(\eta-\sin \eta) \\
l=\left(G M_{G} B^{2}\right)^{1 / 3}(1-\cos \eta) \\
v=\left(\frac{G M_{G}}{B}\right)^{1 / 3} \frac{\sin \eta}{(1-\cos \eta)}
\end{array}
$$

where $\eta$ is a development angle, $l$ is the separation, $v$ is the relative velocity, $t$ is the age of the universe, $B$ is an undetermined coefficient, and $M_{G}$ is the sum of the individual masses (e.g. Peebles 1993). When we observe two clusters on a merger trajectory at the present epoch, we measure the projected separation of he clusters $\Delta l_{\perp}$ and a line of sight velocity differknce $\Delta v_{L O S}$. Modelling the collision as a radial infotI, we parametrize the merger in terms of the angle $\phi$ between the merger trajectory and the line of sight $\left(\Delta v_{L O S}=\Delta v \cos \phi\right.$ and $\left.\Delta l_{\perp}=\Delta l \sin \phi\right)$.

With four free parameters in this model $(\eta, B, \phi$ and $M_{G}$ ) and three observables (the age of the universe $t_{H}$, the projected separation $\Delta l_{\perp}$ and the line of sight velocity difference $\left.\Delta v_{L O S}\right)$, the total cluster mass and age of the universe are a function of the angle $\phi$ and the present epoch value of the development angle $\eta_{H}$.

$$
\begin{array}{r}
M_{G}=2.3 \times 10^{14} M_{\odot}\left(\left(1+\cos \eta_{H}\right) \cos ^{2} \phi \sin \phi\right)^{-1} \\
\left(\frac{\Delta v_{L O S}}{1000 \mathrm{~km} / \mathrm{s}}\right)^{2}\left(\frac{\Delta l_{\perp}}{1 \mathrm{Mpc}}\right) \\
t_{H}=9.8 \times 10^{8} \operatorname{yrs}\left(\frac{\left(\eta_{H}-\sin \eta_{H}\right) \sin \eta_{H}}{\left(1-\cos \eta_{H}\right)^{2}} \cot \phi\right) \\
\left(\frac{\Delta l_{\perp}}{1 \mathrm{Mpc}}\right)\left(\frac{1000 \mathrm{~km} / \mathrm{s}}{\Delta v_{L O S}}\right)
\end{array}
$$

Using an approximate age of the universe to determine $\eta_{H}$, we arrive at the total cluster mass function $M_{G}(\phi)$. Fortunately the dependence of $M_{G}$ on $t_{H}$ is rather weak (see Fig. 5). If the line of sight separation between the subclusters $\Delta l_{L O S}$ is measured, $\phi$ is determined, yielding the total system mass (which we term the cluster merger mass $\left.M_{G}\right) . M_{G}$ can then be compared to other mass estimates, which typically probe the cores of clusters.

Departures from radial infall and the physical extent of the clusters can bias the merger mass. Generally speaking, the large mass and rarity of galaxy clusters make them good candidates for this analysis. We plan to study the accuracy of merger masses using numerical simulations of cluster evolution within "realistic" environments.

Note that there are scenarios in which the merger mass would be substantially biased; it is possible to recognize these scenarios observationally. In the first scenario, the two merging clusters are at small separation ( $\leq 1 \mathrm{Mpc}$ ); interactions among the cluster components, significant overlap of their mass profiles, and small departures from pure radial infall combine to bias the inferred merger velocity and the implied merger mass. Fortunately, the signatures of cluster mergers have been extensively studied (e.g. Geller \& Beers 1982, Dressler \& Shectman 1988, Jones \& Forman 1992, Mohr, Fabricant \& Geller 1993, Pearce, Thomas \& Couchman 1994, Mohr et al. 1995, Buote \& Tsai 1995), and typically there are merger clues in the galaxy and gas distributions.

In the second scenario, a third object of comparable mass to the two merging clusters is close enough to invalidate the two body analysis. In this case, the observed distribution of nearby galaxy clusters should be enough to determine whether or not the radial infall model is appropriate.

\subsection{Merger Mass in A2626}

To calculate $M_{G}$ we require (see Eqn. 4-2) the observed rest frame velocity difference and the projected separation. The rest frame velocity difference is $\Delta v=2,486 \pm 112 \mathrm{~km} / \mathrm{s}$. The projected separation between the centers of mass is assumed to be the distance between the bright, central elliptical in group A (its position is consistent with the peak in the $\mathrm{X}$-ray emission) and the centroid of the 30 group B members with measured redshifts (using the centroid of group A members slightly increases the projected separation and the merger mass). At a cluster distance of $l=175 h^{-1} \mathrm{Mpc}$, the projected separation between the two groups is $\Delta l_{\perp}=(0.707 \pm 0.052) h^{-1} \mathrm{Mpc}$. The uncertainty reflects the statistical uncertainties associated with centroiding group B. The fractional uncertainty in the merger mass $M_{G}$ due to $\Delta v_{L O S}$ 
and $\Delta l_{\perp}$ is $12 \%$.

Fig. 5 displays the ratio of the cluster merger mass $M_{G}$ to the sum of the virial masses of groups A and B $\left(M_{A}+M_{B}=9.1 \times 10^{14} h^{-1} M_{\odot}\right.$; see Table 1 and MGW96) versus the line of sight separation between the subclusters, $\Delta l_{L O S}=\Delta l_{\perp} / \tan \phi$. The dotted line corresponds to $t_{H}=18 \mathrm{Gyr}$ and the solid line corresponds to $t_{H}=13 \mathrm{Gyr}$. The FP distances to galaxies within groups $\mathrm{A}$ and $\mathrm{B}$ yield a ratio of the group B distance to the group A distance $\left(l_{B} / l_{A}\right)$ which can be used to calculate $\Delta l_{L O S}$. Specifically, we estimate the line of sight separation between the subclusters as $\Delta l_{L O S} \sim 175 h^{-1}\left(1-l_{B} / l_{A}\right)$ Mpc. The sample of $24 \mathrm{FP}$ distances to groups $\mathrm{A}$ and $\mathrm{B}$ yields $\Delta l_{L O S}=(14 \pm 18) h^{-1} \mathrm{Mpc}$.

Because distance uncertainties in this case are large, our method does not yield tight mass constraints in Abell 2626; however, if groups A and B are merging (99\% confidence from FP distances and 93\% confidence from galaxy magnitude distributions), it is clear that the virial masses of groups A and B underestimate the total system binding mass. Specifically, the sum of the masses of groups A and B must be at least $1.5 \times 10^{15} M_{\odot}$, compared to the virial sum of $9.1_{-2.7}^{+3.1} \times 10^{14} M_{\odot}$ (MGW96), a factor of 1.65 higher. The virial mass range corresponds to $90 \%$ statistical confidence limits, where it is assumed the mass uncertainties are dominated by the velocity dispersion uncertainties (Heisler, Tremaine \& Bahcall 1985). The virial sum is inconsistent with the minimum merger mass at the $\sim 3 \sigma$ level.

\section{Discussion}

The study of double clusters with the fundamental plane holds promise for significant progress in two areas. First, the study of appropriately isolated nearby double clusters should lead to new constraints on the total cluster binding mass. These constraints will not only provide an independent test of the virial, hydrostatic, and weak lensing mass estimators, but will also provide information about the mass (and typical $M / L$ ) outside the virialized cluster region. Second, the study of more distant double clusters (at $z \geq 0.1$ ) provides a means of directly probing for environmentally driven biases in FP distance estimates.

\subsection{Fundamental Plane Analysis}

Here we apply this approach to the double cluster A2626 at $c z \sim 17,500 \mathrm{~km} / \mathrm{s}$. Because of the cluster distance, it is critical to make seeing corrections while extracting $\theta_{e}$ and $\left\langle\mu_{e}\right\rangle$. Our method is similar to the one described by Saglia et al. (1993b), but includes a seeing-correction uncertainty which makes the photometric parameters less sensitive to the in/exclusion of central points in the profile. We also note that the cosmological dimming correction to the surface brightness has a peculiar velocity dependence which may be important in cases where many FP distance estimates are combined to produce a single, more accurate cluster distance ( $(2.2)$.

Through numerous cross checks we demonstrate that our estimates of the combination $\log \left(\theta_{e}\right)-\beta\left\langle\mu_{e}\right\rangle$ which appears in the FP are sufficiently accurate that their errors make no significant contribution to the FP scatter; using a Monte Carlo approach we estimate the velocity dispersion uncertainties (§2.3). These parameters for the 8 galaxies in group $\mathrm{B}$ and the 16 in group A are listed in Table 2 .

We combine galaxies from both clusters in a single fit, weighting to account for incompleteness (§3.1). Our best fit FP coefficients $(\alpha=1.30 \pm 0.36$ and $\beta=0.31 \pm 0.06)$ are statistically consistent with measurements in other clusters (see Fig. 2). The RMS scatter about this plane in $\Delta \log R_{e}$ is 0.09 , corresponding to $23 \%$ distance uncertainties per galaxy.

We examine the distribution of $M g_{b}$ equivalent widths with the galaxy spectra from both subclusters $(\S 3.2)$. We find that there is no evidence for significant differences in the $M g_{b}-\sigma$ relation (see Fig. 3). Our result does not contradict the recently noted correlation between cluster environment and linestrength (JFK96, Jørgensen 1997); the expected offset in $M g_{b}$ (given the velocity dispersion differences between groups A and B) is too small to detect with our data.

The difference in the fundamental plane zeropoints for the two clusters is $\gamma_{A}-\gamma_{B}=-0.037 \pm 0.046$, consistent with no offset (§3.3). Under the assumption that this zeropoint difference is indicative of distance differences, $\log \left(D_{B} / D_{A}\right)=-0.037 \pm 0.046$, where the uncertainty follows from scatter around the median distance in each cluster with the best fit FP coefficients. We use the $10^{4}$ bootstrap resampling simulations to measure the variation of the distance offset as FP coefficients vary. The relative distance constraint is robust enough to reject the Hubble flow hypothesis $\left(\log \left(D_{B} / D_{A}\right)=0.065\right)$ in $99 \%$ of the simulations. Under the Hubble flow hypothesis, the two clusters are not interacting gravitationally, and the 
velocity difference reflects pure Hubble flow. An analysis of the $R$ band magnitude distributions of confirmed members of both clusters rules out Hubble flow with $93 \%$ confidence (MGW96).

If the subclusters were on an outgoing trajectory (bound or unbound), then the line of sight distance difference would have to be greater than their current recession velocity times the age of the universe $\left(\Delta{ }_{\text {LOS }}>v_{L O S} t_{H} \sim 34 \mathrm{Mpc}\right)$ which is greater than the Hubble flow value: $\log \left(D_{B} / D_{A}\right) \geq 0.0645$; thus, models where groups $\mathrm{A}$ and $\mathrm{B}$ are on outgoing trajectories are ruled out with higher confidence than pure Hubble flow. Therefore, our relative distances indicate that the subclusters are bound and infalling with $99 \%$ confidence (see Fig. A).

\subsection{Radial Infall Model}

Assuming bound and infalling subclusters, we model these kinematics with a radial infall model (Beers, Geller \& Huchra 1982); this two body model is appropriate for separations large compared to the scale of the virialized region of the cluster and in cases where there are no other massive clusters in the neighborhood. Given the projected separation, line of sight velocity difference, and line of sight separation, the radial infall model provides an estimate of the total cluster binding mass which depends weakly on the adopted age of the universe. From Fig. 5, the total binding mass is at least 1.65 times higher than the virial sum, a difference significant at $\sim 3 \sigma$. The total binding mass could be much larger than this minimum.

The differences in these two mass estimates could indicate (1) deviations from radial infall, (2) an error in the virial estimate, or (3) significant mass beyond the virialized region. The $\mathrm{X}$-ray image of A2626 provides no indication of interactions between the two subclusters, and there is no third subcluster, so the radial infall model should be appropriate in A2626. If the virial estimators are biased low, then the radial infall model indicates that $M / L_{R} \sim 1000 h$ (see Table 1), which would be a surprisingly high value (e.g. Ramella et al. 1989). These arguments suggest that the minimum merger mass is larger than the virial mass because it is sensitive to mass in the cluster infall region.

Taking the merger mass as a lower limit on the mass within the cluster infall region, we can address whether there is evidence for variation of the mass to light ratio between the cluster core and infall region. The galaxy light is calculated within a region which only extends to a projected radius of $1.5 h^{-1} \mathrm{Mpc}$; presumably both the galaxy and mass distributions extend beyond this region. We have no CCD photometry over the larger region to directly measure the total cluster light. Assuming that the projected galaxy distribution falls off as $\sim R^{-1.5}$ (e.g. Mohr et al. 1996) with a similar luminosity function to the galaxies in the central region, the total cluster light grows as $\sim \sqrt{R}$ for $R$ larger than the core radius. Under these assumptions, within a diameter of $\sim 4 h^{-1} \mathrm{Mpc}$ there is enough light to yield a mass-tolight ratio of $M / L_{R} \sim 600 h$ throughout the cluster even with the higher mass estimate from the radial infall model. Of course, the binding mass could be many times larger than the minimum value, so the mass-to-light ratio could increase significantly outside the virialized cluster core. A study of the cluster light extending to larger radius and a larger sample of FP distances would yield more concrete information regarding any variation in the cluster mass-to-light ratio in A2626.

FP studies of other double clusters, coupled with (1) a more detailed accounting of the radial distribution of cluster light and (2) larger redshift samples to enable a more accurate estimate of the virial mass, should provide interesting constraints on the distribution of cluster mass and possible differences in the efficiency of galaxy formation beyond the virialized region.

We thank Roberto Saglia and Glenn Baggley for their comments on an earlier version of this manuscript and for their generosity in allowing us to compare our measurements to their unpublished results. We thank Guy Worthey for stimulating discussions, Paul Schechter for obtaining improved $2.4 \mathrm{~m}$ images of several galaxies in our sample, and Emilio Falco for attempting additional imaging. This research was funded in part by NAGW-2367 and NAG5-3401.

\section{REFERENCES}

Abell, G. 1958, ApJS, 3, 211

Baggley, G. 1996, D.Phil. Dissertation, Oxford University

Beers, T.C., Geller, M.J. \& Huchra, J.P. 1982, ApJ, 257, 23

Beers, T.C., Forman, W., Huchra, J.P., Jones, C. \& Gebhardt, K. 1991, AJ, 102, 1581 
Beers, T.C., Gebhardt, K., Huchra, J.P., Forman, W., Jones, C. \& Bothun, G.D. 1992, ApJ, 400, 410

Bender, R., Burstein, D., \& Faber, S.M. 1992, ApJ, 399,462

Bracewell, R.N., 1986, The Fourier Transform and Its Applications, (McGraw-Hill: New York)

Buote, D. \& Tsai, J. 1995, ApJ, 452, 522

Burstein, D., Faber, S., Gaskell, C.M., Krumm, N. 1984, ApJ, 287, 586

Colless, M., Burstein, D., Wegner, G., Saglia, R.P., McMahan, R., Davies, R.L., Bertschinger, E. \& Baggley, G. 1993, MNRAS, 262, 475

Colless, M. \& Dunn, A. 1995, ApJ, 458, 435

David, L.P., Jones, C. \& Forman, W. 1995, ApJ, 445, 578

Djorgovski, S. \& Davis, M. 1987, ApJ, 313, 59

Dressler, A., Lynden-Bell, D., Burstein, D., Davies, R.L., Faber, S.M, Terlevich, R.J. \& Wegner, G. 1987, ApJ, 313, 42

Dressler, A. \& Shectman, S. 1988, AJ, 95, 985

Faber, S.M., Dressler, A., Davies, R.L., Burstein, D., Lynden-Bell, D., Terlevich, R.J. \& Wegner, G. 1987, in Nearly Normal Galaxies, ed. S.M. Faber, (New York: Springer-Verlag), 175

Fabricant, D.G. \& Hertz, E. 1990, SPIE Proc. 1235, 747

Frei, Z. \& Gunn, J.E. 1994, AJ, 108, 1476

Geller, M.J. \& Beers, T.C. 1982, PASP, 94, 421

Guzman, R., Lucey, J.R., Carter, D. \& Terlevich, R.J. 1992, MNRAS, 257, 187

Guzman, R., Lucey, J.R., \& Bower, R.G. 1993, MNRAS, 265, 731

Heisler, J., Tremaine, S. \& Bahcall, J.N. 1985, ApJ, 298, 8

Jackson, R.E. 1982, PhD Dissertation, University of California, Santa Cruz

Jones, C. \& Forman, W. 1992, in Clusters and Superclusters of Galaxies (NATO ASI Vol. 366), ed. A.C. Fabian, London: Kluwer, p. 49

Jørgensen, I. Franx, M. \& Kjærgaard, P. 1993, ApJ, 411, 34 (JFK93)

Jørgensen, I. Franx, M. \& Kjærgaard, P. 1995, MNRAS, 276, 1341

Jørgensen, I. Franx, M. \& Kjærgaard, P. 1996, MNRAS, 280, 167 (JFK96)

Jørgensen, I. 1997, astro-ph 9702076

Lacey, C. \& Cole, S. 1993, MNRAS, 262, 627

Lacey, C. \& Cole, S. 1994, MNRAS, 271, 676

Landolt, A. 1992, AJ, 104, 340

Lucey, J.R. \& Carter, D. 1988, MNRAS, 235, 1177
Lynden-Bell, D., Faber, S.M., Burstein, D., Davies, R.L., Dressler, A., Terlevich, R.J. \& Wegner, G. 1988, ApJ, 326, 19

Mohr, J.J., Fabricant, D.G. \& Geller, M.J. 1993, ApJ, 413,492

Mohr, J.J., Evrard, A.E., Fabricant, D.G. \& Geller, M.J. 1995, ApJ, 447, 8

Mohr, J.J., Geller, M.J., Fabricant, D.G., Wegner, G., Thorstensen, J. \& Richstone, D.O. 1996, ApJ, 470,724

Mohr, J.J., Geller, M.J. \& Wegner, G. 1996, AJ, 112, 1816; (MGW96)

Pearce, F.R., Thomas, P.A. \& Couchman, H.M.P 1994, MNRAS, 268, 953

Peebles, P.J.E. 1993, Principles of Physical Cosmology, (Pinceton University Press: Princeton), p 535

Press, W.H., Flannery, B.P., Teukolsky, S.A. \& Vetterling, W.T. 1988, Numerical Recipes in C, (Cambridge University Press: Cambridge)

Ramella, M., Geller, M.J., \& Huchra, J.P. 1989, ApJ, 344,57

Rose, J.A., Bower, R.G., Caldwell, N., Ellis, R.S., Sharples, R.M. \& Teague, P. 1994, AJ, 108, 2054

Saglia, R.P., Bender, R. \& Dressler, A. 1993a, A\&A, 279, 75

Saglia, R.P., Bertschinger, E., Baggley, G., Burstein, D., Colless, M., Davies, R.L., McMahan, R.K. \& Wegner, G. 1993b, MNRAS, 264, 961

Saglia, R.P., Bertschinger, E., Baggley, G., Burstein, D., Colless, M., Davies, R.L., McMahan, R.K. \& Wegner, G. 1997, MNRAS, submitted

Sandage, A. \& Bedke, J. 1994, The Carnegie Atlas of Galaxies: Volumes I \& II, (Carnegie Institution of Washington: Washington, D.C.)

Savage, B.D. \& Mathis, J.S. 1979, Ann Rev Astron Astro, 17, 73

Scodeggio, M., Solanes, J.M., Giovanelli, R. \& Haynes, M.P. 1995, ApJ, 444, 41

Wegner, G., Colless, M., Baggley, G., Davies, R.L., Bertschinger, E., Burstein, D., McMahan, R.K. \& Saglia, R.P. 1996, ApJS, 106, 1

White, S.D.M., Navarro, J.F., Evrard, A.E. \& Frenk, C.S. 1993, Nature, 366, 429

Worthey, G., Faber, S.M., Gonzalez, J.J. \& Burstein, D. 1994, ApJS, 94, 687

Worthey, G., Trager, S.C. \& Faber, S.M. 1996, in Fresh Views of Elliptical Galaxies, eds. Buzzoni, A., Renzini, A. \& Serrano, A. ASP Conference Series, Vol 86, 203

Worthey, G., \& Ottaviani, D. L. 1997, ApJS, in press

This 2-column preprint was prepared with the AAS LATEX macros $\mathrm{v} 4.0$ 


\section{A. Effects of Culling the Galaxy Sample}

Four of our 24 galaxies show peculiar morphologies; here we detail the peculiarities and demonstrate that eliminating them from the analysis does not significantly alter our conclusions. The following are morphological types which we derived by comparing deep $2.4 \mathrm{~m}$ images and the Carnegie Atlas of Galaxies (Sandage \& Bedke 1994):

- AH: bright nucleus with possible bar; probable SB0

- AL: possible spiral structure; SB0 or Sa

- AM: bright nucleus with possible bar; probable SB0

- AP: appears to have a dust ring; probable RSB0

Without these four galaxies, Group A (B) contains 12 (8) galaxies; we now apply the analysis detailed in $\S 3$ to this smaller sample. The best fit form of the FP is

$$
\log \theta_{e}=(1.39 \pm 0.65) \log \sigma+(0.32 \pm 0.06)\left\langle\mu_{e}\right\rangle+\gamma,
$$

and the scatter around this plane in $\log R_{e}$ is 0.080 (20\% distance uncertainty). These coefficients are consistent with the values from the full sample, but the scatter is somewhat smaller and the $\alpha$ uncertainty is larger. The FP coefficient uncertainties are determined (as before) through bootstrap resampling and refitting the sample.

The RMS scatter around the median distance estimator in group A (B) is $0.066(0.115)$; the zeropoint difference corrected for homogeneous Malmquist bias is

$$
\Delta \gamma=-0.026 \pm 0.045
$$

The zeropoint difference and uncertainty (from the scatter) are somewhat smaller than for the whole sample (see Eqn. 3-4). This measurement is inconsistent with the Hubble flow hypothesis at $\sim 2.0 \sigma$ or $97.7 \%$ confidence. The bootstrap refitting produces a distribution of $\Delta \gamma$ which includes the variation in the FP coefficients; 5000 refits of

this smaller sample rule out the Hubble flow hypothesis with $98.2 \%$ confidence. In summary, removing 4 galaxies which have morphological peculiarities slightly reduces the significance with which the Hubble flow model can be rejected in Abell 2626.

TABLE 1

SubCluster Properties

\begin{tabular}{cccccc}
\hline \hline ID & $\mathrm{N}$ & $\bar{v}$ & $\sigma$ & $M_{v i r}\left[10^{14} M_{\odot}\right]$ & $M_{v i r} / L_{R}$ \\
\hline $\mathrm{A}$ & 67 & $16,533 \pm 141$ & $658_{-81}^{+111}$ & $6.6_{-1.5}^{+2.4}$ & 630 \\
$\mathrm{~B}$ & 30 & $19,164 \pm 138$ & $415_{-72}^{+117}$ & $2.3_{-0.7}^{+1.4}$ & 570 \\
\hline
\end{tabular}

Intervals are statistical $90 \%$ confidence limits 
TABLE 2

Galaxy Properties

\begin{tabular}{lcccccccccc}
\hline \hline Tag & RA(1950) & Decl & $c z$ & $\sigma$ & $\epsilon_{\sigma}$ & $M_{R}$ & $\left\langle\mu_{e}\right\rangle$ & $\theta_{e}^{a}$ & $M g_{b}$ & $F_{B}^{b}$ \\
\hline AA & 233429.30 & 202222.3 & 17,538 & 287 & 12 & 14.06 & 19.79 & 6.232 & 4.83 & 0.53 \\
$\mathrm{AB}$ & 233518.21 & 203156.7 & 17,159 & 223 & 22 & 13.93 & 19.47 & 5.477 & 4.50 & 0.34 \\
$\mathrm{AC}$ & 233422.71 & 205655.6 & 16,819 & 218 & 22 & 14.37 & 19.56 & 4.846 & 4.41 & 0.79 \\
$\mathrm{AD}$ & 233340.11 & 204531.4 & 17,911 & 190 & 22 & 14.75 & 19.93 & 4.817 & 4.64 & 0.63 \\
$\mathrm{AE}$ & 233323.09 & 204202.1 & 15,941 & 205 & 28 & 15.03 & 19.22 & 3.063 & 4.42 & 0.55 \\
$\mathrm{AF}$ & 233533.91 & 210754.7 & 16,728 & 191 & 12 & 14.97 & 19.23 & 3.161 & 4.17 & 0.74 \\
$\mathrm{AG}$ & 233240.07 & 210001.6 & 15,979 & 163 & 19 & 14.71 & 19.33 & 3.727 & 3.83 & 0.85 \\
$\mathrm{AH}$ & 233340.68 & 204235.6 & 16,644 & 161 & 6 & 15.02 & 20.15 & 4.732 & 4.54 & 0.50 \\
$\mathrm{AI}$ & 233432.46 & 201814.5 & 17,804 & 185 & 7 & 14.93 & 19.24 & 3.231 & 4.14 & 0.84 \\
$\mathrm{AJ}$ & 233435.93 & 205941.0 & 16,665 & 178 & 35 & 15.35 & 18.94 & 2.319 & 4.67 & 0.90 \\
$\mathrm{AK}$ & 233358.71 & 210112.4 & 16,856 & 202 & 20 & 15.35 & 19.23 & 2.564 & 4.86 & 0.70 \\
$\mathrm{AL}$ & 233408.75 & 204929.9 & 17,645 & 152 & 3 & 14.61 & 19.67 & 4.554 & 3.67 & 0.39 \\
$\mathrm{AM}$ & 233349.52 & 204912.1 & 16,129 & 252 & 21 & 14.40 & 21.03 & 9.403 & 4.87 & 0.64 \\
$\mathrm{AO}$ & 233357.86 & 205111.8 & 16,113 & 190 & 27 & 15.91 & 17.66 & 0.997 & 4.68 & 1.00 \\
$\mathrm{AP}$ & 233347.81 & 204921.8 & 16,859 & 172 & 8 & 15.07 & 19.70 & 3.743 & 4.86 & 0.30 \\
$\mathrm{AZ}$ & 233519.60 & 203015.3 & 16,063 & 118 & 11 & 16.02 & 19.74 & 2.136 & 3.89 & 0.70 \\
$\mathrm{BA}$ & 233407.96 & 203129.6 & 19,729 & 148 & 3 & 14.84 & 19.19 & 3.351 & 3.80 & 0.69 \\
$\mathrm{BB}$ & 233319.80 & 203312.0 & 19,369 & 219 & 21 & 14.31 & 19.61 & 4.900 & 4.27 & 0.74 \\
$\mathrm{BC}$ & 233357.29 & 203511.2 & 19,240 & 187 & 17 & 14.36 & 20.06 & 6.243 & 4.42 & 0.69 \\
$\mathrm{BD}$ & 233308.31 & 204802.8 & 19,150 & 214 & 10 & 14.21 & 20.48 & 8.148 & 4.45 & 1.00 \\
$\mathrm{BE}$ & 233317.67 & 202254.2 & 19,215 & 133 & 3 & 15.18 & 19.17 & 2.836 & 4.27 & 0.62 \\
$\mathrm{BF}$ & 233333.61 & 204936.5 & 19,825 & 119 & 3 & 15.89 & 19.77 & 2.705 & 3.79 & 0.38 \\
$\mathrm{BG}$ & 233317.50 & 205203.1 & 18,818 & 133 & 6 & 16.02 & 19.71 & 2.431 & 4.61 & 0.68 \\
$\mathrm{BH}$ & 233312.20 & 203520.1 & 19,136 & 151 & 5 & 17.09 & 18.34 & 0.761 & 4.98 & 0.33 \\
\hline
\end{tabular}

${ }^{a}$ in arc seconds

${ }^{\mathrm{b}}$ bulge luminosity fraction 


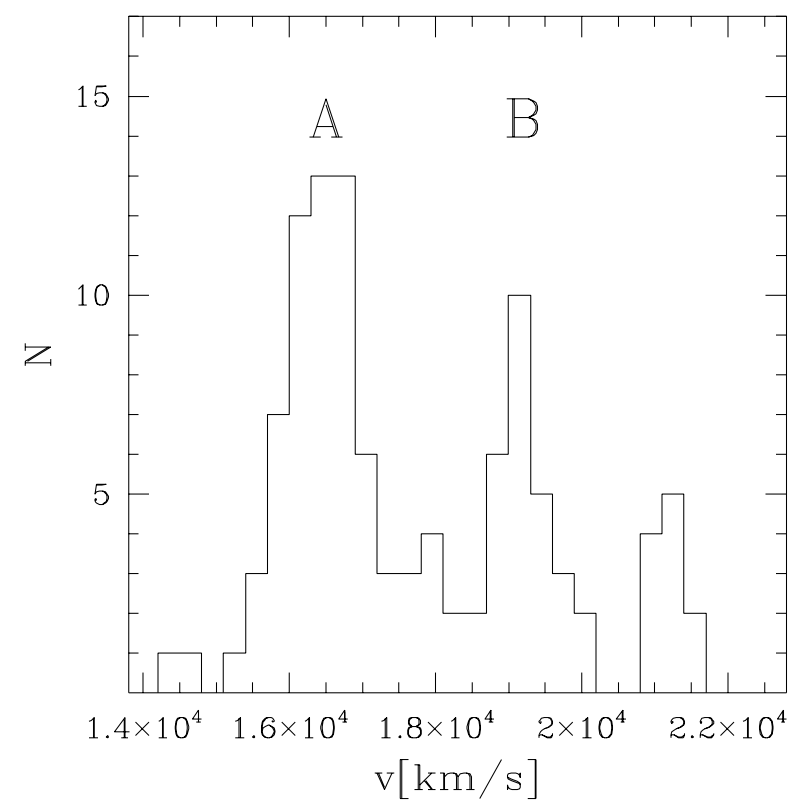

Fig. 1. - The galaxy velocity distribution around Abell 2626. The histogram contains 108 galaxy velocities from the redshift survey of MGW96. Group A is centered at $v=16,500 \mathrm{~km} / \mathrm{s}$, and group B is centered at $v=19,150 \mathrm{~km} / \mathrm{s}$. The high fraction of galaxies with emission lines in the group of 11 centered at $v \sim 21,500 \mathrm{~km} / \mathrm{s}$ ( $73 \%$ compared to $39 \% / 33 \%$ for groups A/B) indicates they are part of a low density background structure. 

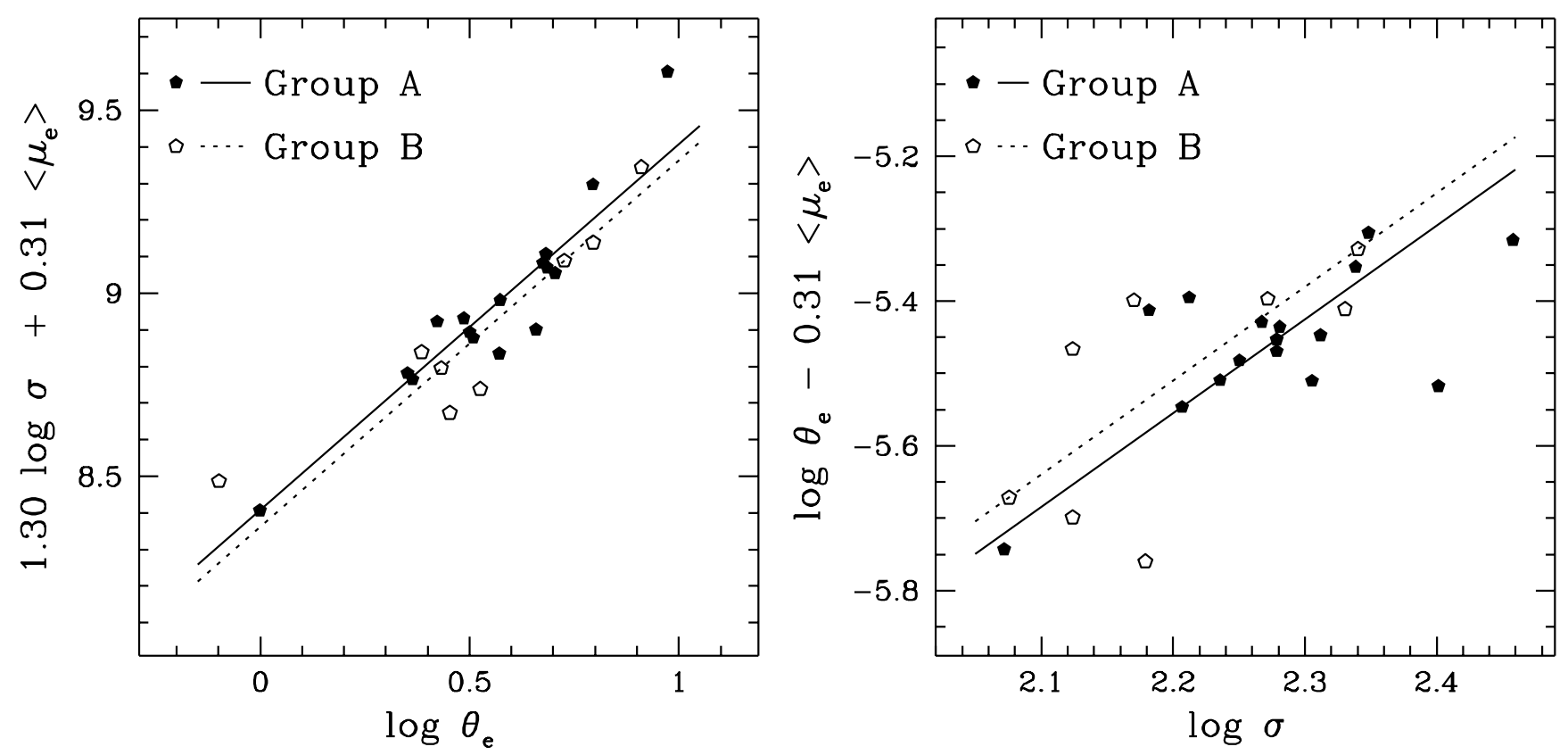

Fig. 2.- Two projections of the FP. Both plots contain group A (solid) and B (hollow) galaxies. The lines define the best fit group A (solid) and B (dashed) FP; offsets in the fits reflect subcluster distance differences. The best fit FP (with weighting to account for incompleteness- see text) is of the form $\log \theta_{e}=1.30 \log \sigma+0.31\left\langle\mu_{e}\right\rangle+\gamma_{c l}$ where $\gamma_{c l}$ is the distance dependent cluster zeropoint. The RMS scatter around the best fit in $\log \theta_{e}$ is 0.09 , corresponding to a $23 \%$ distance uncertainty per galaxy. 


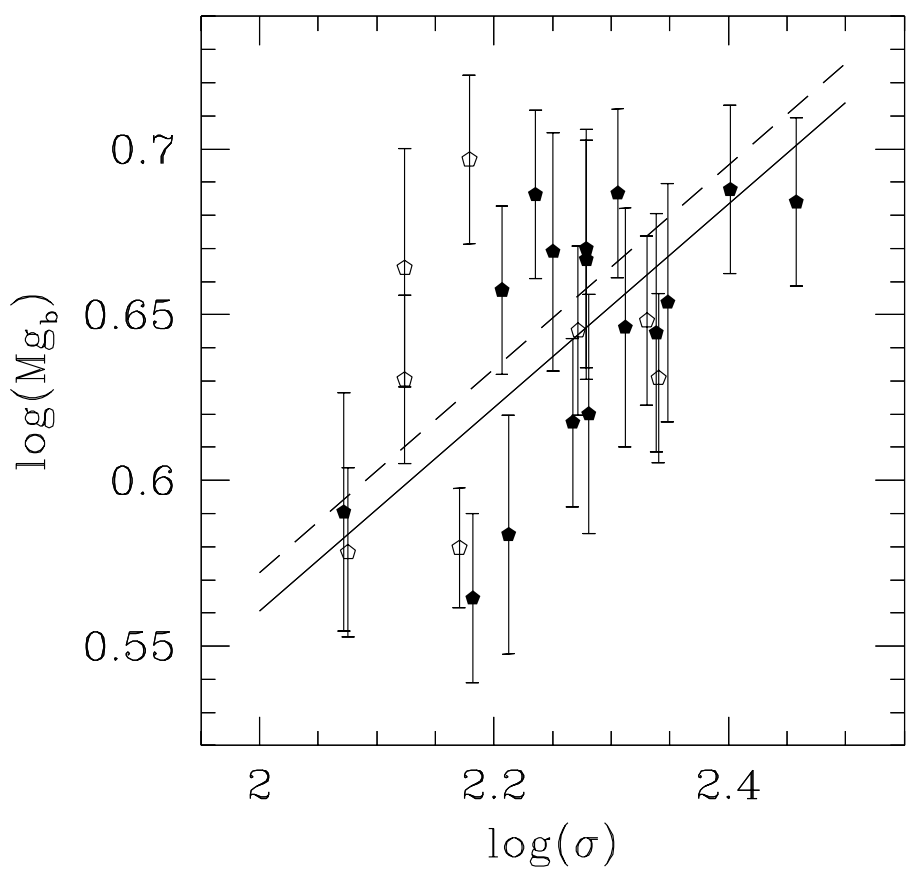

Fig. 3.- Plot of $\log \left(M g_{b}\right)$ vs $\log (\sigma)$ for the galaxies in groups A (solid) and B (hollow). The lines mark the best fit relations for groups A (solid) and B (dashed) constrained to have the slope found using a much larger galaxy sample (Jørgensen 1997). The apparent zeropoint offset between the two samples is statistically insignificant $(0.012 \pm 0.019$; see text), providing no evidence for significant stellar population differences between the subclusters. 


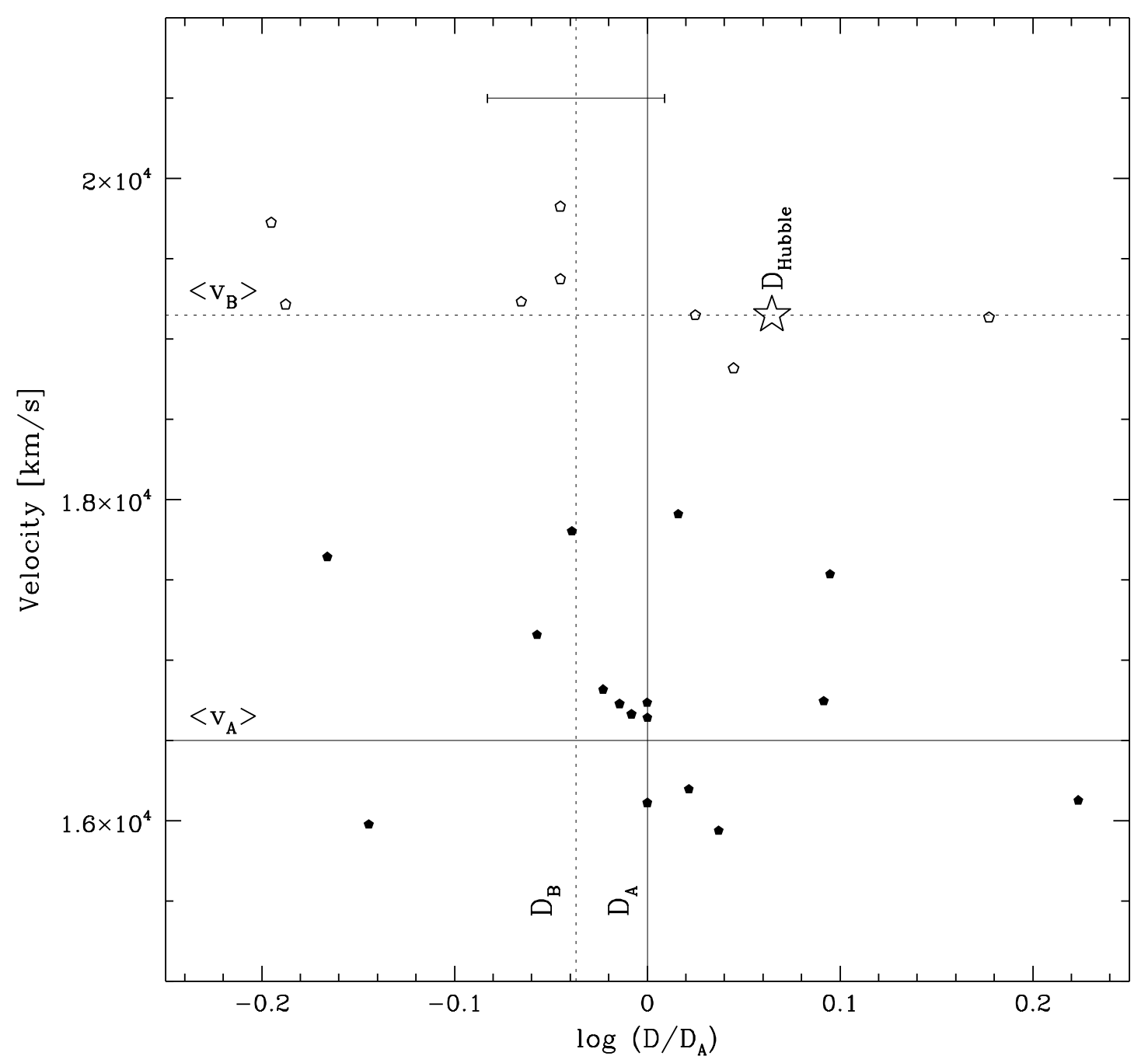

Fig. 4.- Plot of fundamental plane relative distances versus redshift for galaxies in groups A (solid) and B (hollow). Galaxies in groups A and B are clearly offset in velocity (see Fig. 11), and also appear to be offset in distance. Vertical lines mark the (homogeneous Malmquist bias corrected) median distances to groups A (solid) and B (dashed). Horizontal lines mark the mean velocities of groups A (solid) and B (dashed). The large star marked with $\mathrm{D}_{\text {Hubble }}$ lies at the expected position of group $\mathrm{B}$ if its trajectory relative to group $\mathrm{A}$ were pure Hubble flow. The median distances to groups $\mathrm{A}$ and $\mathrm{B}$ indicate that $\log \left(\mathrm{D}_{\mathrm{B}} / \mathrm{D}_{\mathrm{A}}\right)=-0.037 \pm 0.046$; the $1 \sigma$ distance uncertainty is indicated by the error bar on the vertical, dashed line. The sample of 24 galaxies rules out the Hubble flow hypothesis, $\mathrm{D}_{\mathrm{B}}=\mathrm{D}_{\text {Hubble }}$ and $\log \left(\mathrm{D}_{\mathrm{B}} / \mathrm{D}_{\mathrm{A}}\right)=0.065$, with $99 \%$ confidence (see $\S 3.3$ ). 


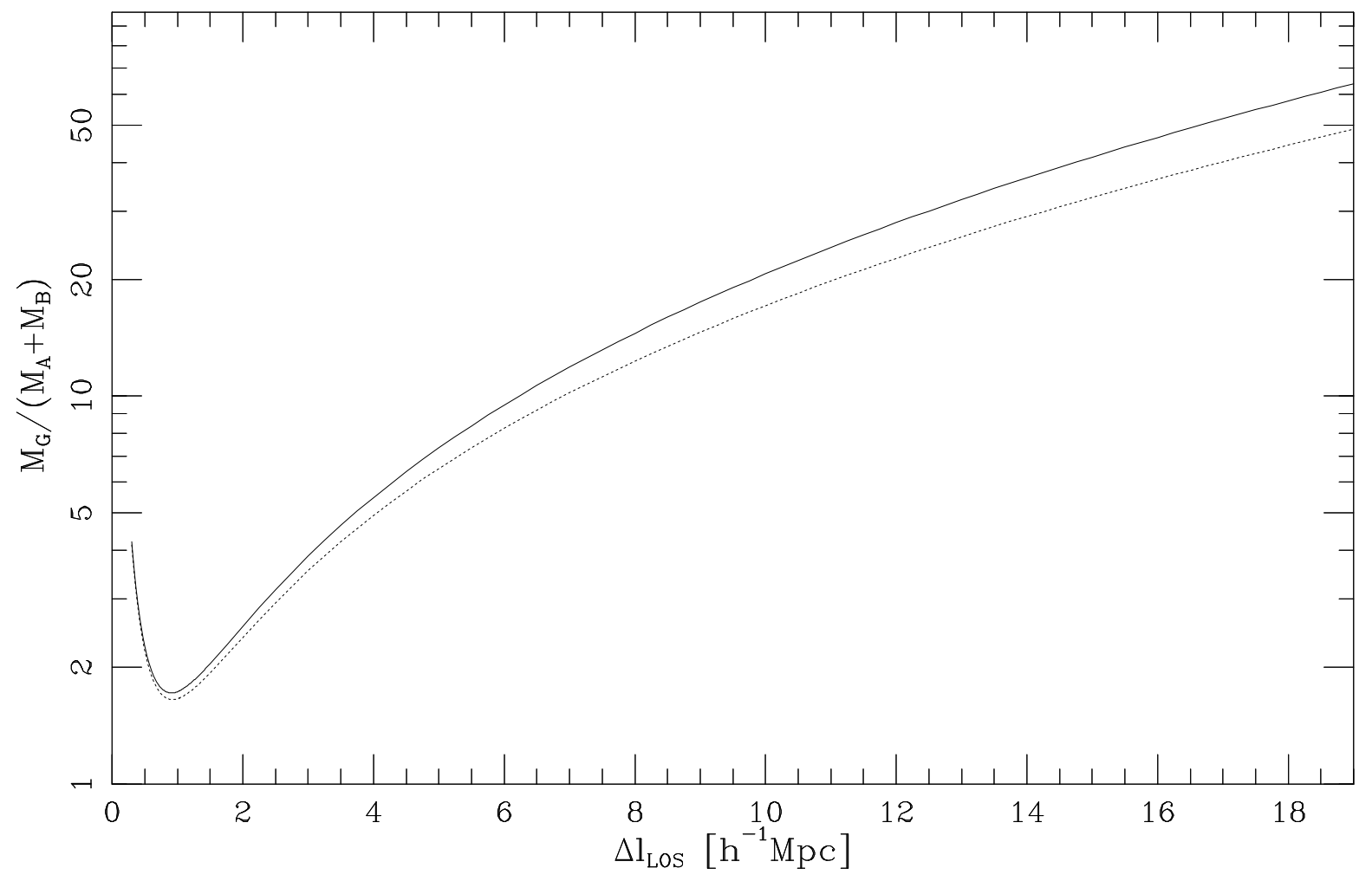

Fig. 5.- The ratio of the cluster merger mass $M_{G}$ to the sum of the group A and B virial masses $M_{A}+M_{B}=$ $9.1 \times 10^{14} h^{-1} M_{\odot}$ as a function of the line of sight separation $\Delta l_{L O S}$ of the two groups. The two solutions correspond to different assumptions regarding the age of the universe $t_{H}: t_{H}=13 \mathrm{Gyr}$ (solid) and $t_{H}=18 \mathrm{Gyr}$ (dotted). The statistical uncertainty in the merger mass $M_{G}$ is $12 \%$. If groups $\mathrm{A}$ and $\mathrm{B}$ are merging, as suggested by the FP distances and the galaxy magnitude distributions, the merger mass is at least 1.65 times greater than the sum of the virial masses. The FP distances indicate that $\Delta l_{L O S}=14 \pm 18 h^{-1} \mathrm{Mpc}$. 